Proceeding Series of the Brazilian Society of Computational and Applied ${ }^{1}$ Mathematics

\title{
Multimodal Energy Harvesting Efficiency Enhancement via Linear Matrix Inequalities Control Driven
}

\section{Douglas da Costa Ferreira ${ }^{1}$}

Department of Mechanical Engineering, Federal University of Mato Grosso, Rondonópolis, MT

\section{Fábio Roberto Chavarette ${ }^{2}$}

Department of Mathematics, UNESP - Univ. Estadual Paulista, Ilha Solteira, SP

\section{Luiz Ricardo Coimbra de Almeida ${ }^{3}$}

Department of Mechanical Engineering, Federal University of Mato Grosso, Rondonópolis, MT

\section{Renato Santos de Souza ${ }^{4}$}

Department of Mechanical Engineering, Federal University of Mato Grosso, Rondonópolis, MT

\section{Luis Paulo Morais Lima ${ }^{5}$}

Department of Mechanical Engineering, Federal University of Mato Grosso, Rondonópolis, MT

\begin{abstract}
There are several solutions to enhance energy harvesting efficiency and is highlighted as the most promised state of the art research the active control driven for increase mechanical energy transformation from vibration to electric energy. The control driven uses the impulse to curt circuit the piezoelectric film and takes advantage of piezoelectric indirect effect. The controller changes the relative stiffness, damping and mass of the studied system and consequently changes the system natural frequency. It is imposed velocity and acceleration to the system to combine the exogenous excitation to system parameters and resulting in maximum mechanical energy transfer. A numeric simulation was lead using a Linear Matrix Inequalities controller applied to a multimodal energy harvester. As main result the controller increased the energy harvester efficiency up to 4 times.
\end{abstract}

Key-words. Energy harvesting, Multimodal, Efficiency, Liner Matrix Inequalities, Controller

\footnotetext{
1 dcferreira@ufmt.br

2 fabioch@mat.feis.unesp.br

3 rick_oceano@hotmail.com

4 syymbolss@yahoo.com.br

${ }^{5}$ luispauloml@gmail.com
} 


\section{Introduction}

Energy harvesting systems are the solutions to convert small source of energy to electric energy [9]. It can be done by wind, solar radiation, radio frequency, small gradient of temperature and vibration [7]. The piezoelectric energy harvesting system are emerging as the better solution as the robustness, transduction capacity and reduced size for small size electronic devices [3]. Although the energy harvesting system are a promise to sustainable energy source their harness are the efficiency because their losses in mechanical energy transduction [1]. The focus of this research is the vibration as exogenous excitation and using a piezoelectric film attached to a cantilever beam connected to an electric load configuring the energy harvesting system.

a)

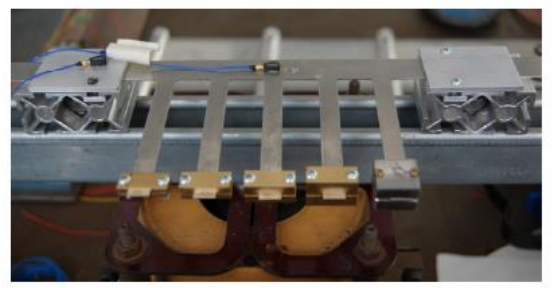

b)

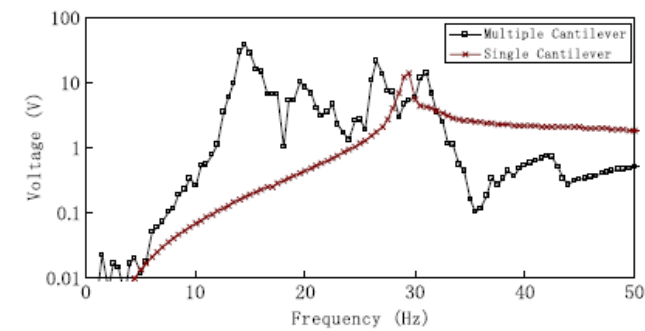

Figure 1: Energy harvesting system configuration [7].

Among several proposal of energy harvesting solutions to enhance their efficiency a multimodal projected was proposed by [8] as shown in Figure 1a. The advantage of using different beams in a multimodal solution is to take advantage of diverse natural frequencies to keep the system in resonance effect in a larger excitation frequency. The Figure 1b shows the output voltage for comparison of single cantilever beam and the multimodal using multiple cantilever beams. Another multimodal solution was studied by [10] and the author concluded that the multimodal solution allow the system to present a resonance behavior along a range varying from 15 to $40 \mathrm{~Hz}$ although a single cantilever beam present resonance only in a narrow range of band around few Hz. A single cantilever beam subjected to a periodic excitation can be described by the dimensionless equation [4]:

$$
\begin{aligned}
\ddot{x}+2 \zeta \dot{x}+\frac{1}{2} x-\chi v & =f \cos \Omega t \\
\dot{v}+\Lambda v+k \dot{x} & =0
\end{aligned}
$$

The equation is dimensionless defined according resonance were $\zeta$ is damping factor, $\chi$ is piezoelectric mechanical coupling coefficient, $\Lambda$ is reciprocal of time constant and $\kappa$ is piezoelectric electric coupling coefficient. According [8] the multimodal solution uses different cantilevers with different natural frequencies. It implies to singular stiffness for specific tip mass and cantilever length. The damping is not changed since the material used for each beam been the same. Also $\chi$ and $\kappa$ are not changed from single to multiple cantilever since it depends on piezoelectric material and $\Lambda$ depends of output load 
capactivity and also is not changed from solutions. Among this many proposals of ${ }^{3}$ multimodal energy harvesting, a two degree of freedom (2DOF) of a spring-mass model can be generalized as shown in Figure 2.

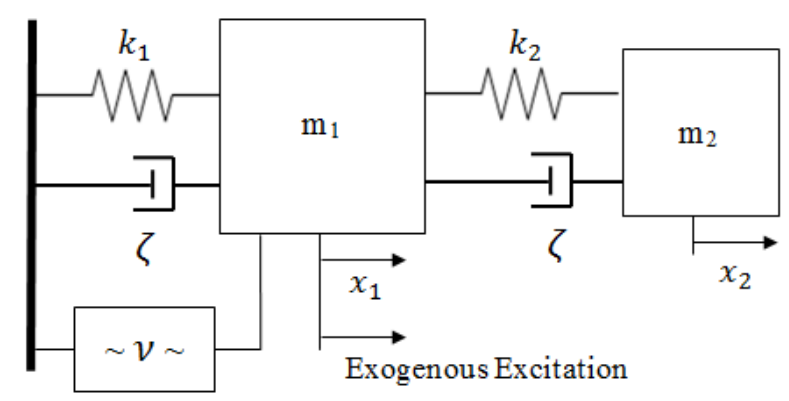

Figure 2: Spring-mass model of a 2DOF energy harvesting.

A proposal to dimensionless by mass mathematic description of a multiple cantilever beam subjected to a periodic excitation, considering $2 \mathrm{DOF}$ is given by:

$$
\begin{gathered}
\ddot{x}_{1}+2 \zeta \dot{x}_{1}+k_{1} x_{1}-k_{2}\left(x_{1}-x_{2}\right)-\chi v=f \cos \Omega t \\
\ddot{x}_{2}+2 \zeta \dot{x}_{2}-k_{2}\left(x_{1}-x_{2}\right)=0 \\
\dot{v}+\Lambda v+k \dot{x}=0
\end{gathered}
$$

Isolating $\ddot{x}$ and $\dot{v}$ and adopting $y_{1}=x_{1}, y_{2}=\dot{x}_{1}, y_{3}=x_{2}, y_{4}=\dot{x}_{2}$ e $y_{5}=v$ the equations in space state are given in Equation 3. Applying Runge-Kutta forth order in equation (4) for dimensionless parameters $k_{1}=0.05, k_{2}=0.03$ (empirically adjusted) and $\zeta=0.01, \Omega=0.8, \chi=0.05, \kappa=0.5, \Lambda=0.05$ and $f=0.083$ [4] and initial conditions $x_{1}(0)=1, x_{2}(0)=0, \dot{x}_{1}(0)=0, \dot{x}_{2}(0)=0$ and $v(0)=0$ it is determined the rate of voltage and time history considering samples from 0 to 2500 in interval of 0.1 totalizing 25,000 time sample according Figure 3a: output voltage. The Figures 3b: phase portrait from mass $\mathrm{m} 1$ and $3 \mathrm{c}$ : phase portrait from mass $\mathrm{m} 2$ considered samples from 2,000 to 2,500 in interval of 0.1 to exclude transient behavior.

$$
\begin{gathered}
\dot{y}_{1}=y_{2} \\
\dot{y}_{2}=-k_{1} y_{1}+k_{2}\left(y_{1}-y_{3}\right)-2 \zeta y_{2}+\chi y_{5}+f \cos \Omega t \\
\dot{y}_{3}=y_{4} \\
\dot{y}_{4}=k_{2}\left(y_{1}-y_{3}\right)-2 \zeta y_{4} \\
\dot{y}_{5}=-\kappa\left(y_{2}-y_{4}\right)-\Lambda y_{5}
\end{gathered}
$$

For the chosen parameters the higher mechanical energy absorption is concentrated in mass $\mathrm{m} 1$ which is connected to the piezoelectric material for output voltage load. For a value of dimensionless stiffness $k_{2}>k_{1}$ the mechanical energy concentration is going to on mass $\mathrm{m} 2$. According system configuration the options for different material with different stiffness can be set for a determined mechanical energy purpose. 
a)

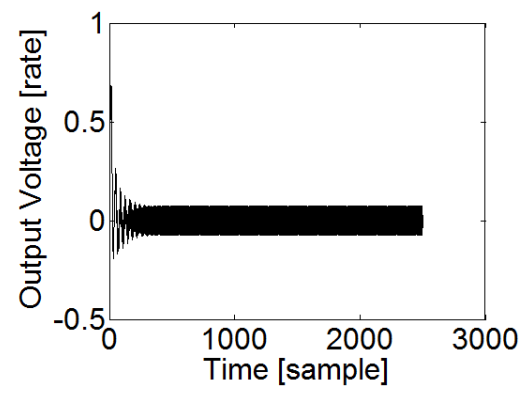

b)

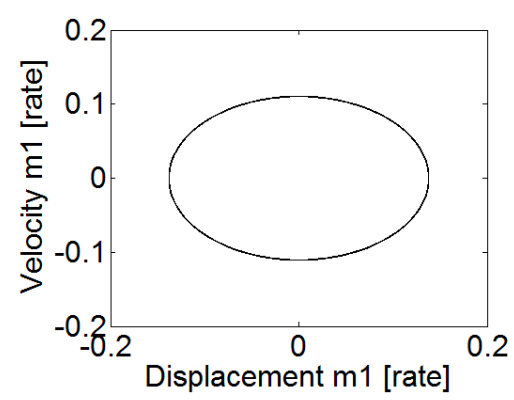

c)

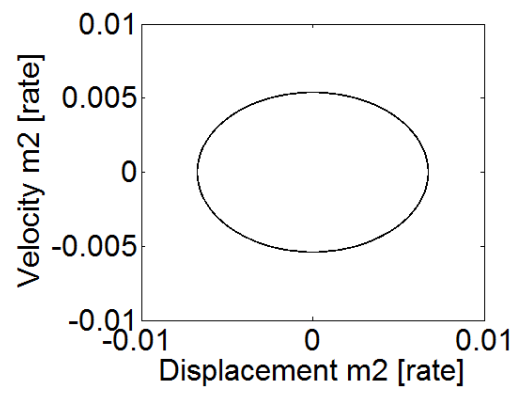

Figure 3: Dynamical behavior of the energy harvesting system.

\section{Controller Definition}

To enhance the mechanical energy transfer from exogenous excitation and the multimodal energy harvesting system there were project a Linear Matrix Inequalities controller (LMI). A controller project to enhance energy harvesting efficiency was proposed by $[5,9]$. Considering the uncertain system given by:

$$
\left\{\begin{array}{l}
\dot{x}=A x+B_{1} w+B_{2} u \\
y=C x
\end{array}\right.
$$

A sufficient condition for the guarantee cost $H \infty$ is the existence of a matrix $X=$ $X^{\prime} \in \mathcal{R}^{n x n}, Y \in \mathcal{R}^{m x n}[2,6]$, since:

with $j=1, \ldots ., \mathrm{r}$

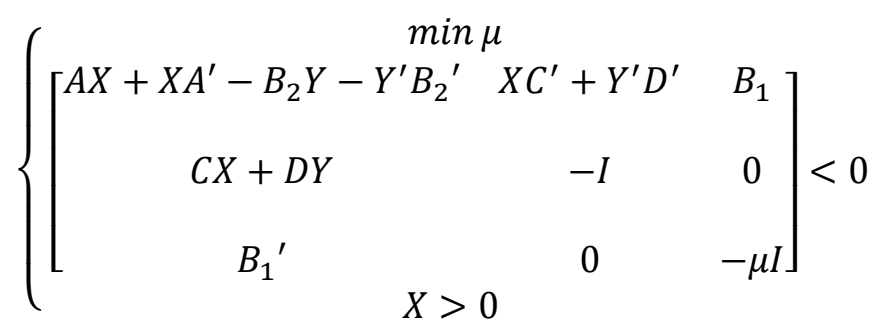

When LMI are feasible a feedback state matrix that stabilize the system is given by $L=Y X^{-1}$. To project the LMI controller the equation 2 is described in matrix form: 


$$
\left\{\begin{array}{l}
\dot{y}_{1} \\
\dot{y}_{2} \\
\dot{y}_{3} \\
\dot{y}_{4} \\
\dot{y}_{5}
\end{array}\right\}=\left[\begin{array}{ccccc}
0 & 1 & 0 & 0 & 0 \\
-k_{1}+k_{2} & -2 \zeta & -k_{2} & 0 & \chi \\
0 & 0 & 0 & 1 & 0 \\
k_{2} & 0 & -k_{2} & -2 \zeta & 0 \\
0 & -\kappa & 0 & \kappa & -\Lambda
\end{array}\right]\left\{\begin{array}{l}
y_{1} \\
y_{2} \\
y_{3} \\
y_{4} \\
y_{5}
\end{array}\right\}+\left[\begin{array}{l}
0 \\
1 \\
0 \\
0 \\
0
\end{array}\right] f \cos \Omega t
$$

Replacing the given parameters in matrix given in equation 5 the LMI matrixes are:

$$
\begin{gathered}
A=\left[\begin{array}{ccccc}
0 & 1 & 0 & 0 & 0 \\
-0.02 & -0.02 & -0.03 & 0 & 0.05 \\
0 & 0 & 0 & 1 & 0 \\
0.03 & 0 & -0.03 & -0.02 & 0 \\
0 & -0.5 & 0 & 0.5 & -0.05
\end{array}\right], B_{1}=\left[\begin{array}{c}
0 \\
0.083 \cos \Omega t \\
0 \\
0 \\
0
\end{array}\right] \\
B_{2}=\left[\begin{array}{l}
1 \\
1 \\
1 \\
1 \\
1
\end{array}\right] e C=\left[\begin{array}{lllll}
1 & 0 & 0 & 0 & 0
\end{array}\right]
\end{gathered}
$$

\section{Efficiency Gain}

Solving the LMI the gain vector is $L=\left[\begin{array}{llll}1.9684 & 5.2764 & -0.3491 & 4.5054\end{array}\right.$ 0.4388]. The controlled system is stable since all eigenvalues have real part negative: $0.1287+0.3302 \mathrm{i} ;-0.1287-0.3302 \mathrm{i} ;-0.0440+0.1804 \mathrm{i} ;-0.0440-0.1804 \mathrm{i} ;-0.0498+0.0000 \mathrm{i}$. Deriving the feedback matrix from the gain vector $L$ it is determined the feedback parameters of control: $\mathrm{k}_{1}=0.0001, \mathrm{k}_{2}=0.0001, \zeta=2.6482, \chi=0.00001, \kappa=5.7764$ and $\Lambda=0.4888$. Replacing feedback parameters of control in the mathematical model of the system and solving the differential equations by applying Runge-Kutta forth order and considering initial conditions $x_{1}(0)=1, x_{2}(0)=0, \dot{x}_{1}(0)=0, \dot{x}_{2}(0)=0$ and $v(0)=0$ it is determined the displacement, velocity and voltage rate considering samples a from 0 to 2,500 in interval of 0.1 totalizing 25,000 time samples. The Figure $4 \mathrm{a}$ and $4 \mathrm{~b}$ shows displacement and velocity of mass $\mathrm{m} 1$. The Figures $4 \mathrm{c}$ and $4 \mathrm{~d}$ shows displacement and velocity of mass $\mathrm{m} 2$. The Figure $4 \mathrm{e}$ shows the system output voltage.

a)

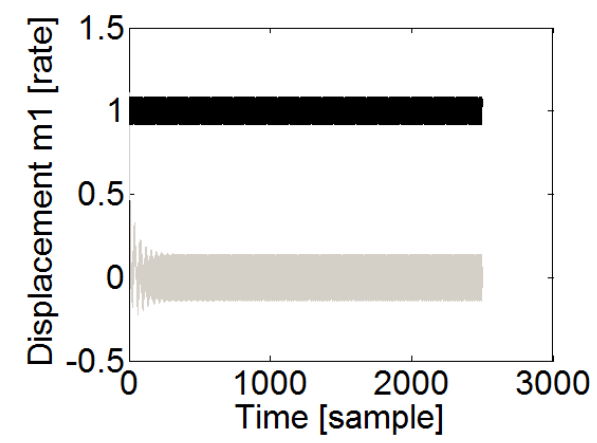

b)

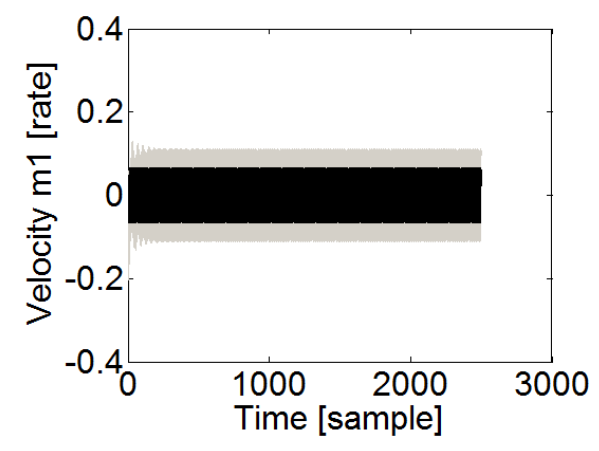


c)

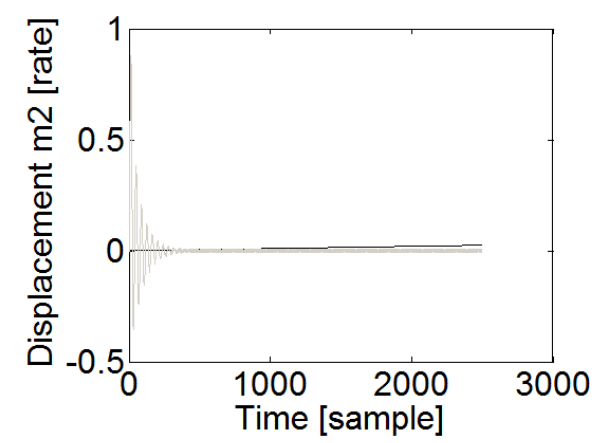

d)

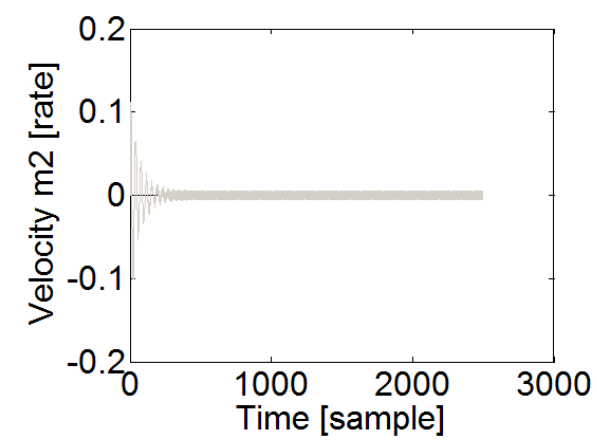

e)

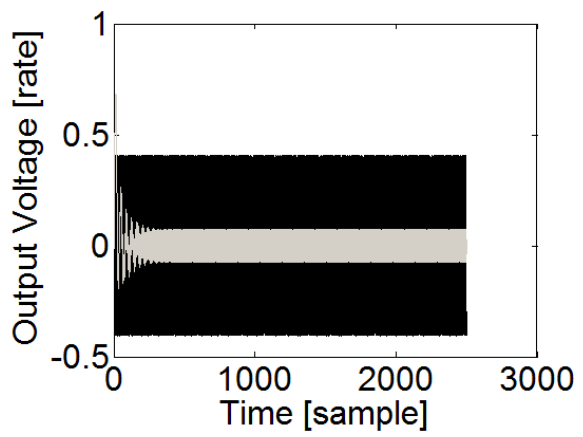

Figure 4: Gray Line - without control; Black Line - Controlled System.

\section{Conclusions}

The LMI controller rises as a promised solution to enhance energy harvesting efficiency for linear mathematical models. In case of multimodal the appliance of a LMI to enhance energy harvesting efficiency appears as a novel solution. The overall gain of energy in terms of output voltage is shown in Table 1.

Table 1: Overall gain for active control.

\begin{tabular}{|c|c|}
\hline & RMS voltage rate \\
\hline Without control & 0.0692 \\
\hline Active LMI Controller & 0.2862 \\
\hline Gain & 4.136 \\
\hline
\end{tabular}

It is presented a gain up to 4 times which represent a promise solution to enhance efficiency in multimodal energy harvesting system.

\section{Acknowledgements}

The authors would like to thanks to Federal University of Mato Grosso, UNESP Univ. Estadual Paulista, Postdoctoral in Mechanical Engineering program for support. To Fundação de Amparo a Pesquisa do Estado de São Paulo (FAPESP Process No. 
2014/16807-3), Conselho Nacional de Desenvolvimento Científico e Tecnológico ${ }^{7}$ (CNPq Process No. 301769/2012-5) and to Fundação de Amparo a Pesquisa do Estado de Mato Grosso (FAPEMAT) for scholarship and funding.

\section{References}

[1] B. Andò, S. Baglio, C. Trigona, N. Dumas, L. Latorre, P. Nouet, Nonlinear mechanism in MEMS devices for Energy Harvester applications, Journal of Micromechanics and Microengineering, v.20, n. 125020, 1-12 (2010), DOI: $10.1088 / 0960-1317 / 20 / 12 / 125020$.

[2] M. Chilali, P. Gahinet, P. Hळ design with pole placement constrains: an LMI approach, IEEE Transactions on Automatic Control, v. 41, n. 3, 358-367 (1996), 0018-9286/96.

[3] Z. Dibin, Vibration energy harvester: machinery vibration; human movement and flow induced vibration, Sustainable Energy Harvester Technologies, 25-54 (2011).

[4] A. Erturk and D. J. Inman, Broadband piezoelectric power generation on highenergy orbits of the bistable duffing oscillator with electromechanical coupling, Journal of Sound and Vibration, v. 330, n. 10, 2339-2353 (2011), DOI: 10.1016/j.jsv.2010.11.018.

[5] D. C. Ferreira, F. R. Chavarette, N. J. Peruzzi, Optimal Linear Control Driven for Piezoelectric Non-Linear Energy Harvesting on Non-Ideal Excitation Sourced, Advanced Materials Research, Vols. 971-973, 1107-1112 (2014), DOI: 10.4028/www.scientific.net/AMR.971-973.1107.

[6] R. A. T. Mendes, E. Assunção, M. C. M. Teixeira, C. Q. Andrea, Dynamic tracking with zero variation and disturbance rejection applied to discrete-time systems, Mathematical Problems in Engineering, v. 842896, 1-20 (2010), DOI: $10.1155 / 2010 / 842896$.

[7] V. Raghunathan, A. Kansal, J. Hsu, J. Friedman, M. Srivastava, Design considerations for solar energy harvesting wireless embedded systems, IEEE, 457462 (2005), 0-7803-9201-9.

[8] S. Qi, R. Shuttleworth, S. O. Oyadiji, J. Wright, Design of a multiresonant beam for broadband piezoelectric energy harvesting, Smart Mater. Struct., vol. 19, 1-10 (2010), DOI: 10.1088/0964-1726/19/9/094009.

[9] H. A. Sodano, and D. J. Inman, A review of power harvesting from vibration using piezoelectric materials, The Shock and Vibration Digest, v. 36, n. 3, 197-205 (2004), LA-UR-03-5397.

[10] L. Tang, Y. Yang, A multiple-degree-of-freedom piezoelectric energy harvesting model. Journal of Intelligent Material Systems and Structures, v. 23, 1631-1647 (2012), DOI: 10.1177/1045389X12449920. 